\title{
CLASSIFICATION OF ROTATIONAL SURFACES IN PSEUDO-GALILEAN SPACE
}

\author{
DAE WON YOON \\ Gyeongsang National University, South Korea
}

\begin{abstract}
In the present paper, we study rotational surfaces in the three dimensional pseudo-Galilean space $G_{3}^{1}$. Also, we characterize rotational surfaces in $G_{3}^{1}$ in terms of the position vector field, Gauss map and Laplacian operator of the second fundamental form on the surface.
\end{abstract}

\section{INTRODUCTION}

Let $M$ be a connected $n$-dimensional submanifold of the $m$-dimensional Euclidean space $\mathbb{E}^{m}$, equipped with the induced metric. Denote by $\Delta$ the Laplacian of $M$ acting on smooth functions on $M$. Takahashi ([17]) classified the submanifolds in $\mathbb{E}^{m}$ in terms of an isometric immersion $\mathbf{x}$ and the Laplacian of $M$. He proved that $M$ satisfying $\Delta \mathbf{x}=\lambda \mathbf{x}$, that is, all coordinate functions are eigenfunctions of the Laplacian with the same eigenvalue $\lambda \in \mathbb{R}$ are either the minimal submanifolds of $\mathbb{E}^{m}$ or the minimal submanifolds of hypersphere $\mathbb{S}^{m-1}$ in $\mathbb{E}^{m}$. As a generalization of Takahashi's theorem for the case of hypersurfaces, Garay ([8]) considered the hypersurfaces in $\mathbb{E}^{m}$ whose coordinate functions are eigenfunctions of the Laplacian, that is, he studied the hypersurfaces satisfying the condition

$$
\Delta \mathbf{x}=A \mathbf{x},
$$

where $A \in \operatorname{Mat}(m, \mathbb{R})$ is an $m \times m$ - diagonal matrix.

2010 Mathematics Subject Classification. 53A35, 53C25.

Key words and phrases. Pseudo-Galilean space, rotational surface, second fundamental form.

This paper was supported by Basic Science Research Program through the National Research Foundation of Korea(NRF) funded by the Ministry of Education, Science and Technology(2012R1A1A2003994). 
On the other hand, the study of an isometric immersion satisfying (1.1) can be extended to Gauss map on a hypersurface of Euclidean space. The Gauss map is a useful tool to examine the character of the hypersurfaces in Euclidean space. In [6], Dillen, Pas and Verstraelen studied the surfaces of revolution in Euclidean 3 -space $\mathbb{E}^{3}$ such that its Gauss map $G$ satisfies the condition

$$
\Delta G=A G
$$

where $A \in \operatorname{Mat}(3, \mathbb{R})$ is a $3 \times 3$-real matrix. Several geometers have studied surfaces satisfying the conditions (1.1) and (1.2) in the ambient spaces, and many interesting results have been obtained in $[1-4,18,19]$ etc.

If a surface $M$ in the ambient spaces has a non-degenerate second fundamental form $I I$ or a non-degenerate third fundamental form $I I I$, then it is regarded as a new (pseudo-)Riemannian metric on $M$. So, considering the conditions (1.1) and (1.2), we may have a natural question as follows: What are the surfaces in the ambient spaces satisfying the conditions

$$
\Delta^{p} \mathbf{x}=A \mathbf{x}
$$

$$
\Delta^{p} G=A G
$$

where $\Delta^{p}$ is the Laplacian with respect to $p$ of $M, p=I I$ or III and $A \in$ $\operatorname{Mat}(3, \mathbb{R})$ ?

Several results for the above question were obtained, when the ambient spaces are the Euclidean space $([11])$ and the Minkowski space $([5,9,10,12$, $14,15]$.

The main purpose of this paper is to complete classification of rotational surfaces in the three dimensional pseudo-Galilean space $G_{3}^{1}$ satisfying (1.3) and (1.4) with $p=I I$.

\section{Preliminaries}

The pseudo-Galilean geometry is one of the real Cayley-Klein geometries (of projective signature $(0,0,+,-)$, explained in [13]). The absolute of the pseudo-Galilean geometry is an ordered triple $\{\omega, f, I\}$, where $\omega$ is the ideal (absolute) plane, $f$ the line in $\omega$ and $I$ the fixed hyperbolic involution of $f$.

In affine coordinates defined by $\left(x_{0}: x_{1}: x_{2}: x_{3}\right)=(1: x: y: z)$, the distance between the points $P_{i}=\left(x_{i}, y_{i}, z_{i}\right)(i=1,2)$ is defined by (cf. [16])

$$
d\left(P_{1}, P_{2}\right)=\left\{\begin{array}{lr}
\left|x_{2}-x_{1}\right|, & \text { if } x_{1} \neq x_{2} \\
\sqrt{\left|\left(y_{2}-y_{1}\right)^{2}-\left(z_{2}-z_{1}\right)^{2}\right|}, & \text { if } x_{1}=x_{2}
\end{array}\right.
$$


The group motions of $G_{3}^{1}$ is a six-parameter group given (in affine coordinates) by

$$
\begin{aligned}
& \bar{x}=a+x, \\
& \bar{y}=b+c x+y \cosh \varphi+z \sinh \varphi, \\
& \bar{z}=d+e x+y \sinh \varphi+z \cosh \varphi .
\end{aligned}
$$

Let $\mathbf{x}=\left(x_{1}, y_{1}, z_{1}\right)$ and $\mathbf{y}=\left(x_{2}, y_{2}, z_{2}\right)$ be vectors in $G_{3}^{1}$. A vector $\mathbf{x}$ is called isotropic if $x_{1}=0$, otherwise it is called nonisotropic. The pseudoGalilean scalar product of $\mathbf{x}$ and $\mathbf{y}$ is defined by

$$
\langle\mathbf{x}, \mathbf{y}\rangle= \begin{cases}x_{1} x_{2}, & \text { if } x_{1} \neq 0 \quad \text { or } \quad x_{2} \neq 0, \\ y_{1} y_{2}-z_{1} z_{2}, & \text { if } x_{1}=0 \text { and } x_{2}=0 .\end{cases}
$$

From this, the pseudo-Galilean norm of a vector $\mathbf{x}$ in $G_{3}^{1}$ is given by $\|\mathbf{x}\|=$ $\sqrt{|\langle\mathbf{x}, \mathbf{x}\rangle|}$ and all unit nonisotropic vectors are the form $\left(1, y_{1}, z_{1}\right)$. There are four types of isotropic vectors: spacelike $\left(y_{1}^{2}-z_{1}^{2}>0\right)$, timelike $\left(y_{1}^{2}-z_{1}^{2}<0\right)$ and the two types of lightlike $\left(y_{1}= \pm z_{1}\right)$ vectors. A non-lightlike isotropic vector is a unit vector if $y_{1}^{2}-z_{1}^{2}= \pm 1$.

The pseudo-Galilean cross product of $\mathbf{x}$ and $\mathbf{y}$ on $G_{3}^{1}$ is defined by

$$
\mathbf{x} \times \mathbf{y}=\left|\begin{array}{ccc}
0 & -e_{2} & e_{3} \\
x_{1} & y_{1} & z_{1} \\
x_{2} & y_{2} & z_{2}
\end{array}\right|
$$

where $e_{2}=(0,1,0)$ and $e_{3}=(0,0,1)$.

Consider a $C^{r}$-surface $M, r \geq 1$, in $G_{3}^{1}$ parametrized by

$$
\mathbf{x}\left(u_{1}, u_{2}\right)=\left(x\left(u_{1}, u_{2}\right), y\left(u_{1}, u_{2}\right), z\left(u_{1}, u_{2}\right)\right) \text {. }
$$

Let us denote $g_{i}=\frac{\partial x}{\partial u_{i}}, h_{i j}=\left\langle\frac{\partial \tilde{\mathbf{x}}}{\partial u_{i}}, \frac{\partial \tilde{\mathbf{x}}}{\partial u_{j}}\right\rangle(i, j=1,2)$, where $\sim$ stands for the projection of a vector on the pseudo-Euclidean $y z$-plane. A surface $M$ is called admissible if it does not have Euclidean tangent planes. Therefore a surface $M$ is admissible if and only if $x_{, i} \neq 0$ for some $i=1,2$.

Let $M$ be an admissible surface. Then the unit normal vector field $U$ of a surface $M$ is defined by

$$
U=\frac{1}{W}\left(0, x_{, 1} z_{, 2}-x_{, 2} z_{, 1}, x_{, 1} y_{, 2}-x_{, 2} y_{, 1}\right),
$$

where

$$
W=\sqrt{\left|\left(x_{, 1} y_{, 2}-x_{, 2} y_{, 1}\right)^{2}-\left(x_{, 1} z_{, 2}-x_{, 2} z_{, 1}\right)^{2}\right|} .
$$

On the other hand, the matrix of the first fundamental form $d s^{2}$ of a surface $M$ in $G_{3}$ is given by ([16])

$$
d s^{2}=\left(\begin{array}{cc}
d s_{1}^{2} & 0 \\
0 & d s_{2}^{2}
\end{array}\right),
$$

where $d s_{1}^{2}=\left(g_{1} d u_{1}+g_{2} d u_{2}\right)^{2}$ and $d s_{2}^{2}=h_{11} d u_{1}^{2}+2 h_{12} d u_{1} d u_{2}+h_{22} d u_{2}^{2}$. Here $g_{i}=x_{, i}$ and $h_{i j}=\left\langle\tilde{\mathbf{x}}_{, i}, \tilde{\mathbf{x}}_{, j}\right\rangle(i, j=1,2)$. In such case, we denote the 
coefficients of $d s^{2}$ by $g_{i j}^{*}$. A surface is spacelike or timelike if the determinant of the matrix $\left(g_{i j}^{*}\right)$ is positive or negative, respectively.

The coefficients $L_{i j}, i, j=1,2$ of the second fundamental form $I I$, which are the normal components of $\mathbf{x}_{, i, j}, i, j=1,2$, that is,

$$
L_{i j}=\frac{1}{g_{1}}\left\langle g_{1} \tilde{\mathbf{x}}_{, i, j}-g_{i, j} \tilde{\mathbf{x}}_{, 1}, U\right\rangle=\frac{1}{g_{2}}\left\langle g_{2} \tilde{\mathbf{x}}_{, i, j}-g_{i, j} \tilde{\mathbf{x}}_{, 2}, U\right\rangle .
$$

If a surface $M$ in $G_{3}^{1}$ has a non-degenerate second fundamental form $I I$, then it is regarded as a new (pseudo-)Riemannian metric on $(M, I I)$. Let $\left\{u_{1}, u_{2}\right\}$ be a local coordinate system of $M$ and $L_{i j}(i, j=1,2)$ be the coefficients of the second fundamental form $I I$ on $M$. We denote by $\left(L^{i j}\right)$ (resp. $\mathfrak{L})$ the inverse matrix (resp. the determinant) of the matrix $\left(L_{i j}\right)$. Then, the Laplacian $\Delta^{I I}$ of the second fundamental form $I I$ on $M$ is defined by

$$
\Delta^{I I}=-\frac{1}{\sqrt{|\mathfrak{L}|}} \sum_{i, j=1}^{2} \frac{\partial}{\partial u_{i}}\left(\sqrt{|\mathfrak{L}|} L^{i j} \frac{\partial}{\partial u_{j}}\right) .
$$

\section{Rotational SuRfaCes in $G_{3}^{1}$}

In the pseudo-Galilean space $G_{3}^{1}$ we distinguish between two types of circles and between two types of rotational surfaces. The first type occurs as the result of a pseudo-Euclidean rotation and the second as the result of an isotropic rotation. It is well-known that the normal form of pseudo-Euclidean rotations is given by

$$
\begin{aligned}
& \bar{x}=x, \\
& \bar{y}=y \cosh t+z \sinh t, \\
& \bar{z}=y \sinh t+z \cosh t
\end{aligned}
$$

and the normal form of isotropic rotations is given by

$$
\begin{aligned}
& \bar{x}=x+b t, \\
& \bar{y}=y+x t+b \frac{t^{2}}{2}, \\
& \bar{z}=z,
\end{aligned}
$$

where $t \in \mathbb{R}$ and $b=$ constant $>0$ (cf. [16]).

The trajectory of a single point under a pseudo-Euclidean rotation is a pseudo-Euclidean circle (i.e., a rectangular hyperbola)

$$
x=\text { constant }, \quad y^{2}-z^{2}=r^{2}, \quad r \in \mathbb{R} .
$$

The invariant $r$ is the radius of the circle. Pseudo-Euclidean circles intersect the absolute line $f$ in the fixed points of the hyperbolic involution $\left(F_{1}, F_{2}\right)$. There are three kinds of pseudo-Euclidean circles: circles of real radius, of imaginary radius, and of radius zero. Circles of real radius are timelike curves 
(having timelike tangent vectors) and imaginary radius spacelike curves (having spacelike tangent vectors).

The trajectory of a point under an isotropic rotation is an isotropic circle whose normal form is

$$
z=\text { constant, } \quad y=\frac{x^{2}}{2 b} \text {. }
$$

The invariant $b$ is the radius of the circle. The fixed line of the isotropic rotation (3.2) is the absolute line $f$.

Let $\alpha$ be a plane curve and $l$ a given line. It is convenient, but not necessary, to start with a plane curve $\alpha$. A rotational surface is a surface obtained when $\alpha$ is displaced in a pseudo-Euclidean rotation or an isotropic rotation about $l([7])$.

First of all, we consider a nonisotropic curve $\alpha$ parameterized by

$$
\alpha(u)=(f(u), g(u), 0) \quad \text { or } \quad \alpha(u)=(f(u), 0, g(u))
$$

around the $x$-axis by the pseudo-Euclidean rotation (3.1), where $g$ is a positive function and $f$ is a smooth function on an open interval $I$. Then the rotational surface $M$ can be written as

$$
\mathbf{x}(u, v)=(f(u), g(u) \cosh v, g(u) \sinh v),
$$

or

$$
\mathbf{x}(u, v)=(f(u), g(u) \sinh v, g(u) \cosh v),
$$

for any $v \in \mathbb{R}$, which is called a nonisotropic rotational surface.

Next, we consider the isotropic rotations. By an isotropic curve $\alpha(u)=$ $(0, f(u), g(u))$ about the $z$-axis by an isotropic rotation (3.2), we obtain a surface

$$
\mathbf{x}(u, v)=\left(v, f(u)+\frac{v^{2}}{2 b}, g(u)\right)
$$

where $f$ and $g$ are smooth functions and $b \neq 0$. This surface is called an isotropic rotational surface.

\section{Rotational SURFACES SATisfying $\Delta^{I I} \mathbf{x}=A \mathbf{x}$}

In this section, we classify rotational surfaces in $G_{3}^{1}$ satisfying the condition

$$
\Delta^{I I} \mathbf{x}=A \mathbf{x}
$$

where $A=\left(a_{i j}\right), i, j=1,2,3$.

First of all, let $M$ be a rotational surface in $G_{3}^{1}$ defined by (3.3). Assume that the rotated curve $\alpha$ is parameterized by arc-length, that is,

$$
\alpha(u)=(u, g(u), 0) .
$$


In this case, the parametrization of $M$ is given by

$$
\mathbf{x}(u, v)=(u, g(u) \cosh v, g(u) \sinh v)
$$

where $g$ is a positive function.

From now on, we shall often not write the parameter $u$ explicitly in our formulas. By (2.3), the coefficients of the second fundamental form $I I$ on $M$ are obtained by

$$
L_{11}=g^{\prime \prime}, L_{12}=0, L_{22}=g .
$$

Here the prime denotes the derivative with respect to $u$. Since the surface has a non-degenerate second fundamental form $I I$, the functions $g$ and $g^{\prime \prime}$ are non-vanishing everywhere. By a straightforward computation, the Laplacian $\Delta^{I I}$ of the second fundamental form $I I$ on $M$ can be expressible as

$$
\Delta^{I I}=-\frac{1}{2 g^{\prime \prime}}\left(\frac{g^{\prime}}{g}-\frac{g^{\prime \prime \prime}}{g^{\prime \prime}}\right) \frac{\partial}{\partial u}-\frac{1}{g^{\prime \prime}} \frac{\partial^{2}}{\partial u^{2}}-\frac{1}{g} \frac{\partial^{2}}{\partial v^{2}} .
$$

Suppose that $M$ satisfies (4.1). Then, from (4.2) and (4.4), we have the following system of differential equations:

$$
\begin{aligned}
-\frac{1}{2 g^{\prime \prime}}\left(\frac{g^{\prime}}{g}-\frac{g^{\prime \prime \prime}}{g^{\prime \prime}}\right) & =a_{11} u+a_{12} g \cosh v+a_{13} g \sinh v \\
\left(-\frac{g^{\prime}}{2 g^{\prime \prime}}\left(\frac{g^{\prime}}{g}-\frac{g^{\prime \prime \prime}}{g^{\prime \prime}}\right)-2\right) \cosh v & =a_{21} u+a_{22} g \cosh v+a_{23} g \sinh v \\
\left(-\frac{g^{\prime}}{2 g^{\prime \prime}}\left(\frac{g^{\prime}}{g}-\frac{g^{\prime \prime \prime}}{g^{\prime \prime}}\right)-2\right) \sinh v & =a_{31} u+a_{32} g \cosh v+a_{33} g \sinh v
\end{aligned}
$$

From (4.5) we easily deduce that $a_{12}=a_{13}=a_{21}=a_{23}=a_{31}=a_{32}=0$ and $a_{22}=a_{33}$, that is, the matrix $A$ is diagonal. We put $a_{11}=\lambda$ and $a_{22}=a_{33}=\mu$. Then the system (4.5) reduces now to the following equations

$$
\begin{gathered}
g^{\prime} g^{\prime \prime}-g g^{\prime \prime \prime}=-2 \lambda u g g^{\prime 2}, \\
g^{\prime}\left(g^{\prime} g^{\prime \prime}-g g^{\prime \prime \prime}\right)=2 \mu g^{2} g^{\prime{ }^{2}}+4 g g^{\prime \prime 2} .
\end{gathered}
$$

Combining two equations in (4.6) we get

$$
\lambda u g^{\prime}+\mu g+2=0
$$

Thus its general solution is either

$$
g(u)=-\frac{2}{\lambda} \ln u+c_{1}
$$

if $\lambda \neq 0$ and $\mu=0$, or

$$
g(u)=\frac{1}{\mu}\left(c_{1} u^{-\frac{\mu}{\lambda}}-2\right)
$$

if $\lambda \neq 0$ and $\mu \neq 0$, where $c_{1}$ is constant. The remained cases with respect to $\lambda$ and $\mu$ are do not appear. Consequently, we have 
THEOREM 4.1. Let $M$ be a nonisotropic rotational surface generated by a curve $\alpha(u)=(u, g(u), 0)$ in the three dimensional pseudo-Galilean space $G_{3}^{1}$. If $M$ satisfies the condition (4.1), then $M$ is a timelike surface and parameterized as

$$
\mathbf{x}(u, v)=(u, g(u) \cosh v, g(u) \sinh v)
$$

where

(1) either $g(u)=-\frac{2}{\lambda} \ln u+c_{1}$,

(2) or $g(u)=\frac{1}{\mu}\left(c_{1} u^{-\frac{\mu}{\lambda}}-2\right)$ with $\lambda \neq 0, \mu \neq 0, c_{1} \in \mathbb{R}$.

Remark. For the specific constants $\lambda, \mu, c_{1}$ of (1) and (2) in Theorem 4.1, we have the graphs shown in Figure 1 and Figure 2, respectively.

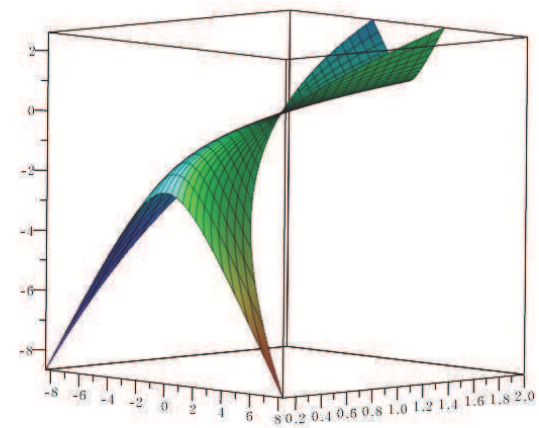

FiguRE 1. A nonoisotopic rotational surface with $g(u)=\ln u$.

Let $M$ be a nonisotropic rotational surface given by (3.4) in the three dimensional pseudo-Galilean space $G_{3}^{1}$. Assume that a nonisotropic curve $\alpha$ is a unit speed curve, that is, $\alpha(u)=(u, 0, g(u))$. In the case, the surface $M$ is parameterized by

$$
\mathbf{x}(u, v)=(u, g(u) \sinh v, g(u) \cosh v) .
$$

Suppose that the surface $M$ satisfies (4.1). Then, by using the similar method of Theorem 4.2, we can find the same equation (4.7). Consequently, we have

THEOREM 4.2. Let $M$ be a nonisotropic rotational surface given by (4.10) in the three dimensional pseudo-Galilean space $G_{3}^{1}$. If $M$ satisfies the condition (4.1), then $M$ is a spacelike surface and parameterized as

$$
\mathbf{x}(u, v)=(u, g(u) \sinh v, g(u) \cosh v),
$$




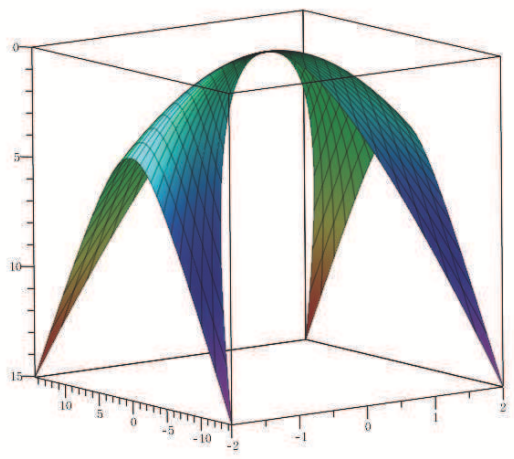

Figure 2. A nonoisotopic rotational surface with $g(u)=$ $u^{2}-2$.

where

(1) either $g(u)=-\frac{2}{\lambda} \ln u+c_{1}$,

(2) or $g(u)=\frac{1}{\mu}\left(c_{1} u^{-\frac{\mu}{\lambda}}-2\right)$ with $\lambda \neq 0, \mu \neq 0, c_{1} \in \mathbb{R}$.

Next, we consider rotational surfaces $M$ in $G_{3}^{1}$ generated by an isotropic curve $\alpha(u)=(0, f(u), g(u))$. Assume that the parameter $u$ is the arc-length parameter of $\alpha$, that is,

$$
f^{\prime}(u)^{2}-g^{\prime}(u)^{2}=-\epsilon(= \pm 1) .
$$

Then the parametrization of $M$ is given by

$$
\mathbf{x}(u, v)=\left(v, f(u)+\frac{v^{2}}{2 b}, g(u)\right),
$$

where $f$ and $g$ are smooth functions and $b \neq 0$.

On the other hand, the coefficients of the second fundamental form $I I$ on $M$ are obtained by

$$
L_{11}=\epsilon\left(f^{\prime} g^{\prime \prime}-f^{\prime \prime} g^{\prime}\right), L_{12}=0, L_{22}=\frac{\epsilon}{b} g^{\prime} .
$$

Since $\mathfrak{L}=-\frac{\epsilon}{b} f^{\prime \prime}$, the function $f^{\prime \prime}$ is non-vanishing everywhere. From this and (4.11), the function $g^{\prime}$ is also non-vanishing everywhere. By (4.11) we find $g^{\prime \prime}=\left(f^{\prime} / g^{\prime}\right) f^{\prime \prime}$, it follows that $L_{11}=-f^{\prime \prime} / g^{\prime}$. Thus the Laplacian $\Delta^{I I}$ of $M$ is easily obtained by

$$
\Delta^{I I}=-\frac{1}{f^{\prime \prime}}\left(g^{\prime \prime}-\frac{f^{\prime \prime \prime} g^{\prime}}{2 f^{\prime \prime}}\right) \frac{\partial}{\partial u}-\frac{g^{\prime}}{f^{\prime \prime}} \frac{\partial^{2}}{\partial u^{2}}+\frac{\epsilon b}{g^{\prime}} \frac{\partial^{2}}{\partial v^{2}} .
$$


Equation (4.1) is equivalent to an expression

$$
\begin{aligned}
0 & =a_{11} v+a_{12}\left(f+\frac{v^{2}}{2 b}\right)+a_{13} g, \\
-\frac{f^{\prime}}{f^{\prime \prime}}\left(g^{\prime \prime}-\frac{f^{\prime \prime \prime} g^{\prime}}{2 f^{\prime \prime}}\right)-g^{\prime}+\epsilon \frac{1}{g^{\prime}} & =a_{21} v+a_{22}\left(f+\frac{v^{2}}{2 b}\right)+a_{23} g, \\
-\frac{g^{\prime}}{f^{\prime \prime}}\left(g^{\prime \prime}-\frac{f^{\prime \prime \prime} g^{\prime}}{2 f^{\prime \prime}}\right)-\frac{g^{\prime} g^{\prime \prime}}{f^{\prime \prime}} & =a_{31} v+a_{32}\left(f+\frac{v^{2}}{2 b}\right)+a_{33} g .
\end{aligned}
$$

We can easily find $a_{11}=a_{12}=a_{13}=a_{21}=a_{22}=a_{31}=a_{32}=0$. In this case, (4.15) is rewritten as the following:

$$
\begin{aligned}
-\frac{f^{\prime}}{f^{\prime \prime}}\left(g^{\prime \prime}-\frac{f^{\prime \prime \prime} g^{\prime}}{2 f^{\prime \prime}}\right)-g^{\prime}+\epsilon \frac{1}{g^{\prime}} & =a_{23} g, \\
-\frac{g^{\prime}}{f^{\prime \prime}}\left(g^{\prime \prime}-\frac{f^{\prime \prime \prime} g^{\prime}}{2 f^{\prime \prime}}\right)-\frac{g^{\prime} g^{\prime \prime}}{f^{\prime \prime}} & =a_{33} g .
\end{aligned}
$$

If we multiply the first equation of (4.16) by $g^{\prime}$ and the second equation of (4.16) by $-f^{\prime}$, and add the resulting equations, we get $a_{33} f^{\prime}=a_{23} g^{\prime}$. Up to a rigid motion, there exists a real number $k \in \mathbb{R}$ such that $g(u)=k f(u)$. Consequently, the following theorem holds.

THEOREM 4.3. Let $M$ be an isotropic rotational surface generated by an isotropic curve in the three dimensional pseudo-Galilean space $G_{3}^{1}$. If $M$ satisfies the condition (4.1), then, for any smooth function $f(u), M$ is parameterized as

where $k$ is constant.

$$
\mathbf{x}(u, v)=\left(v, f(u)+\frac{v^{2}}{2 b}, k f(u)\right)
$$

\section{Rotational SURFACES SATiSfying $\Delta^{I I} G=A G$}

In this section, we classify rotational surfaces in $G_{3}^{1}$ satisfying the condition

$$
\Delta^{I I} G=A G,
$$

where $A=\left(a_{i j}\right), i, j=1,2,3$.

First, we consider a nonisotropic rotational surface $M$ in $G_{3}^{1}$ generated by a curve $\alpha(u)=(u, g(u), 0)$. Then the parametrization of $M$ is given by

$$
\mathbf{x}(u, v)=(u, g(u) \cosh v, g(u) \sinh v)
$$

where $g$ is a positive function. For the nondegeneracy of the second fundamental of $M$, we assume that $g^{\prime \prime}$ is nonvanishing everywhere. From (2.2) the Gauss map $G$ of $M$ is obtained by

$$
G=\left(\frac{1}{\left\|\mathbf{x}_{u} \times \mathbf{x}_{v}\right\|}\right) \mathbf{x}_{u} \times \mathbf{x}_{v}=(0, \cosh v, \sinh v) .
$$


Then, the Laplacian $\Delta^{I I} G$ of the Gauss map $G$ together with (4.4) and (5.3) gives

$$
\Delta^{I I} G=-\frac{1}{g} G
$$

THEOREM 5.1. Let $M$ be a nonisotropic rotational surface given by (5.2) in the three dimensional pseudo-Galilean space $G_{3}^{1}$. Then the Gauss map $G$ of $M$ satisfies (5.4).

If a nonisotropic rotational surface $M$ satisfies (5.1), then the function $g$ is constant. It is a contradiction. Thus, we have

THEOREM 5.2. There is no nonisotropic rotational surfaces given by (5.2) satisfying (5.1) in the three dimensional pseudo-Galilean space $G_{3}^{1}$.

On a nonisotropic rotational surface given by (4.10), we can also obtain the following result:

THEOREM 5.3. Let $M$ be a nonisotropic rotational surface given by (4.10) in the three dimensional pseudo-Galilean space $G_{3}^{1}$. Then the following statements hold:

(1) The Gauss map $G$ of $M$ satisfies $\Delta^{I I} G=\frac{1}{g} G$.

(2) There is no the surface $M$ satisfying (5.1) in $G_{3}^{1}$.

Next, let $M$ be an isotropic rotational surface generated by a unit speed isotropic curve in $G_{3}^{1}$. Then $M$ is parameterized by

$$
\mathbf{x}(u, v)=\left(v, f(u)+\frac{v^{2}}{2 b}, g(u)\right) .
$$

From this the Gauss map $G$ of $M$ is given by

$$
G=\left(0,-g^{\prime}(u),-f^{\prime}(u)\right) .
$$

Suppose that $M$ satisfies (5.1). Then from (4.14) and (5.6) we get the following system of differential equations:

$$
\begin{gathered}
a_{12} g^{\prime}(u)+a_{13} f^{\prime}(u)=0, \\
-a_{22} g^{\prime}(u)-a_{23} f^{\prime}(u)=\frac{1}{f^{\prime \prime}}\left(\frac{1}{2} f^{\prime} f^{\prime \prime \prime}+f^{\prime \prime 2}\right), \\
-a_{32} g^{\prime}(u)-a_{33} f^{\prime}(u)=\frac{1}{f^{\prime \prime}}\left(\frac{1}{2} f^{\prime \prime \prime} g^{\prime}+f^{\prime \prime} g^{\prime \prime}\right) .
\end{gathered}
$$

If we multiply (5.8) by $g^{\prime 2}$ and (5.9) by $-f^{\prime} g^{\prime}$, and add the resulting equations, we obtain

$$
\epsilon f^{\prime \prime}=\left(a_{32}-a_{23}\right) f^{\prime}\left({f^{\prime}}^{2}+\epsilon\right)+\left(\left(a_{33}-a_{22}\right) f^{\prime 2}-a_{22} \epsilon\right) g^{\prime} .
$$


Thus, (5.7) and (5.10) imply

$$
\lambda f^{\prime \prime}+\mu f^{\prime 3}+\nu f^{\prime}=0,
$$

where

$$
\begin{aligned}
& \lambda=a_{12}, \\
& \mu=\epsilon\left(a_{13}\left(a_{33}-a_{22}\right)-a_{12}\left(a_{32}-a_{23}\right)\right), \\
& \nu=a_{12}\left(a_{23}-a_{32}\right)-a_{13} a_{22} .
\end{aligned}
$$

Then a general solution of this equation is

$$
f(u)= \pm \frac{\lambda}{\sqrt{|\mu \nu|}} \arctan \left(\frac{\sqrt{\left|e^{\frac{2 \nu}{\lambda}\left(u+d_{1}\right)}-\mu\right|}}{\sqrt{|\mu|}}\right)+d_{2} .
$$

From (5.7) and (5.12), the function $g(u)$ becomes

$$
g(u)=\mp \frac{\delta}{\sqrt{|\mu \nu|}} \arctan \left(\frac{\sqrt{\left|e^{\frac{2 \nu}{\lambda}\left(u+d_{1}\right)}-\mu\right|}}{\sqrt{|\mu|}}\right)+d_{3},
$$

where $\delta=a_{13}$ and $d_{1}, d_{2}, d_{3}$ are constants of integration. The surface generated by (5.12) and (5.13) is showed in Figure 3.

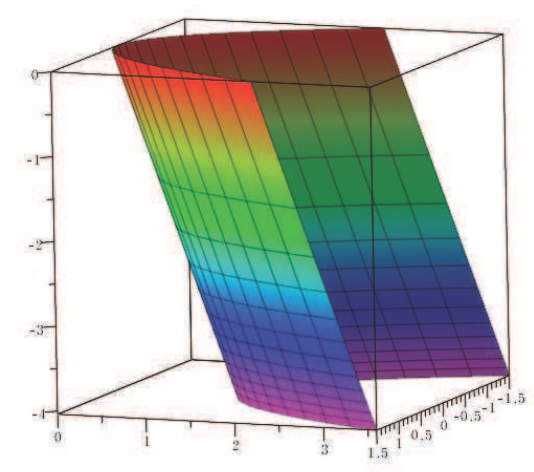

Figure 3. A isotropic rotational surface with $f(u)=$ $\arctan \left(\sqrt{e^{2 u}-1}\right)$ and $g(u)=-3 f(u)$.

TheOREM 5.4. An isotropic rotational surface in the three dimensional pseudo-Galilean space $G_{3}^{1}$ satisfies the condition $\Delta^{I I} G=A G$ if and only if 
the surface is a sphere given by

$$
\mathbf{x}(u, v)=\left(v, f(u)+\frac{v^{2}}{2 b}, g(u)\right),
$$

where $f(u)= \pm \frac{\lambda}{\sqrt{|\mu \nu|}} \arctan \left(\frac{\sqrt{\left|e^{\frac{2 \nu}{\lambda}\left(u+d_{1}\right)}-\mu\right|}}{\sqrt{|\mu|}}\right)+d_{2}$ and $g(u)=-\frac{\delta}{\lambda} f(u)$.

\section{ACKNOWLEDGEMENT.}

The author wishes to express their sincere thanks to the referee for making several useful comments.

\section{REFERENCES}

[1] L. J. Alías, A. Ferrández and P. Lucas, Surfaces in the 3-dimensional LorentzMinkowski space satisfying $\Delta x=A x+B$, Pacific J. Math. 156 (1992), 201-208.

[2] L. J. Alías, A. Ferrández, P. Lucas and M. A. Meroño, On the Gauss map of B-scrolls, Tsukuba J. Math. 22 (1998), 371-377.

[3] C. Baikoussis and D. E. Blair, On the Gauss map of ruled surfaces, Glasgow Math. J. 34 (1992), 355-359.

[4] S. M. Choi, On the Gauss map of surfaces of revolution in a 3-dimensional Minkowski space, Tsukuba J. Math. 19 (1995), 351-367.

[5] M. Choi, Y. H. Kim and D. W. Yoon, Some classification of surfaces of revolution in Minkowski 3-space, J. Geom. 104 (2013), 85-106.

[6] F. Dillen, J. Pas and L. Vertraelen, On the Gauss map of surfaces of revolution, Bull. Inst. Math. Acad. Sinica 18 (1990), 239-246.

[7] B. Divjak and Ž. Milin Šipuš, Some special surface in the pseudo-Galilean space, Acta Math. Hungar. 118 (2008), 209-226.

[8] O. J. Garay, An extension of Takahashi's theorem, Geom. Dedicata 34 (1990), 105112.

[9] G. Kaimakamis and B, Papantoniou, Surfaces of revolution in the 3-dimensional Lorentz-Minkowski space satisfying $\Delta^{I I} \vec{r}=A \vec{r}$, J. Geom. 81 (2004), 81-92.

[10] G. Kaimakamis, B. Papantoniou and K. Petoumenos, Surfaces of revolution in the 3-dimensional Lorentz-Minkowski space $\mathbb{E}_{1}^{3}$ satisfying $\Delta^{I I I} \vec{r}=A \vec{r}$, Bull. Greek Math. Soc. 50 (2005), 75-90.

[11] Y. H. Kim, C. W. Lee and D. W. Yoon, On the Gauss map of surfaces of revolution without parabolic points, Bull. Korean Math. Soc. 46 (2009), 1141-1149.

[12] C. W. Lee, Y. H. Kim and D. W. Yoon, Ruled surfaces of non-degenerate third fundamental forms in Minkowski 3-spaces, Appl. Math. Comput. 216 (2010), 32003208.

[13] E. Molnár, The projective interpretation of the eight 3-dimensional homogeneous geometries, Beiträge Algebra Geom. 38 (1997), 261-288.

[14] B. Senoussi and M. Bekkar, Helicoidal surfaces in the three-dimensional LorentzMinkowski space satisfying $\Delta^{I I} r=A r$, Kyushu J. Math. 67 (2013), 327-338.

[15] B. Senoussi and M. Bekkar, Helicoidal surfaces in the 3-dimensional LorentzMinkowski space $\mathbb{E}_{1}^{3}$ satisfying $\Delta^{I I I} r=A r$, Tsukuba J. Math. 37 (2013), 339-353.

[16] Ž. Milin Šipuš and B. Divjak, Surfaces of constant curvature in the pseudo-Galilean space, Int. J. Math. Math. Sci. 2012, Art. ID 375264, 28 pp.

[17] T. Takahashi, Minimal immersions of Riemannian manifolds, J. Math. Soc. Japan 18 (1966), 380-385. 
[18] D. W. Yoon, On the Gauss map of translation surfaces in Minkowski 3-space, Taiwanese J. Math. 6 (2002), 389-398.

[19] D. W. Yoon, Surfaces of revolution in the three dimensional pseudo-Galilean space, Glas. Mat. Ser. III 48(68) (2013), 415-428.

Dae Won Yoon

Department of Mathematics Education and RINS

Gyeongsang National University

Jinju 660-701

South Korea

E-mail: dwyoon@gnu.ac.kr

Received: 16.6 .2014$.

Revised: 23.2 .2015 . \& 1.6.2015. 